\title{
Microgels and fractal structures at interfaces and surfaces
}

\author{
Thomas A. Vilgis and Michael Stapper \\ Max-Planck-Institut für Polymerforschung, Postfach 3148, 55021 Mainz, Germany
}

(April 20, 2022)

\begin{abstract}
The behavior of microgels near surfaces and their adsorption is studied by simple scaling theory. Two different types of microgels can be studied, i.e., fractal type microgels and randomly crosslinked polymer chains. In the first case the gel can be described mainly by introducing a spectral dimension. The second type requires more attention and uses the number of crosslinks as parameter. The main result is that soft gels with weakly coupled crosslinks and a low number of crosslinks adsorb much better than hard gels, with many crosslinks. Similar results for fractal gels and branched polymer are presented. Fractal gels with low connectivity adsorb easier than gels with a large connectivity dimension. We discuss also consequences on surface protection by microgels.
\end{abstract}

PACS: $36.20 \mathrm{Ey}, 82.70 . \mathrm{Gg}$,

\section{INTRODUCTION}

The theory of polymers near surfaces is a very important subject for theoretical investigation. The main reason is the very broad and deep theoretical research possible for these types of systems. Modern theories have been developed and brought to a very high standard [1]. On the other hand there is a large demand on practical interest on studying polymers near surfaces. One of them is surface protection. The theory on polymer brushes [2] is a typical example of these type of applications. Polymers are attached to surfaces to protect the surface from further adsorption of, e.g., biological active molecules such as proteins. These molecules need to be pretended to from all kind of surface interactions in order to save their biological function [3]. The (technical) problem with polymer brushes is, that a large surface coverage is needed to protect the surface in a most effective way. Polymers attached chemically or physically at one end at the surface form brushes, i.e., the chains are extended and the brush height $h$ follows a scaling law $h \simeq \sigma^{-1 / 3} N$ (in good solvent), where $\sigma$ is the area per chain an is thus related to the grafting density. $N$ the degree of polymerization of the chains. The problem is to set up a small value of $\sigma$, or, correspondingly a large grafting density. For low values of the grafting density the chains behave as "mushrooms" and the surface protection is incomplete.

In earlier papers it has been shown by one of us, that the use of branched chains in much more effective [4 6 . Chains, polymers and polymeric fractals with a larger connectivity seem to be more appropriate to protect surfaces more effectively. Indeed due to their connectivity their occupied area is larger and it turns out that these systems behave more like single chain fractals. Typical many body effects, such as occur in conventional polymer brushes do not play a significant role.

In the present paper we suggest a different route of surface protection by using microgels and branched structure. Microgels had become an important tool in designing polymeric nano structures. These systems can be synthesized 77-10 with different structures. Indeed the structure of these microgels can range from a fractal state, i.e., a branched self-similar polymer with a large connectivity, up to almost hard and highly crosslinked spheres. Such systems are well designed to study the transition form polymer to colloid behavior by variation of the structure and the crosslinking state.

We have shown earlier [11,12], that fractal polymers and gels can interpenetrates each other and screen excluded volume forces, wherever their connectivity is low. In terms of the spectral dimension this is the case, when it is lower than a critical value, i.e. $D_{\mathrm{c}}=6 / 5$. Thus fractals with lower spectral dimensions screen their excluded volume, whereas polymeric fractals with larger spectral dimension saturate. Then they form soft balls, which cannot interpenetrate each other and are well separated from each other [11,12]. This state has an analogon in the case of linear polymers. Polymer melts in two dimensions correspond to a saturated state. The individual chains are separated from each other and form on average disks on a hexagonal lattice 13 .

For surface protection in three dimensions it would thus be more effective to use fractal microgels with a large connectivity. Then the surface coverage is ruled by the single gel behavior. Alternatively crosslinked gels can be used to have the same effect. Sufficiently crosslinked gels cannot interpenetrate each other. The adsorption behavior can be studied then by the single gel adsorption. The significant parameters are either the connectivity (spectral dimension $D$ ) or the crosslink number $M$. In the following we emphasize mainly on the crosslinked gels rather on the self similar connected polymeric fractals. Nevertheless we will consider both cases below.

The paper is organized as follows. In section III we introduce the model of self crosslinked polymer chains, which form the microgels. In section III we repeat briefly the scaling behavior of ideal microgels before we consider in section 
IV the effects of excluded volume in the bulk. Sections $\mathrm{V}$ and VI treat the adsorption behavior of the gels close to attracting walls using simple scaling arguments. In section $\mathrm{V}$ we will also make some remarks on fractal type gels.

\section{THE MODEL}

Flexible interacting macromolecule modeled usually by the Edwards Hamiltonian in three dimensional space. The Edwards Hamiltonian consists of two parts, i.e.,the Gaussian connectivity of monomers

$$
H_{\mathrm{W}}=\frac{3}{a^{2}} \sum_{i=1}^{N}\left(\mathbf{R}_{i}-\mathbf{R}_{i-1}\right)^{2}
$$

and the self avoidance between monomers

$$
H_{\mathrm{I}}=v \sum_{0 \leq i \leq j}^{N} \delta\left(\mathbf{R}_{i}-\mathbf{R}_{j}\right)
$$

Thereby $v>0$ is the excluded volume of the monomers, $N$ the degree of polymerization, and $a$ the Kuhn length. The chain configurations are determined by monomer coordinates $\mathbf{R}_{i}$, where $i$ labels all monomers $1 \leq i \leq N$. The Edwards Hamiltonian is sufficient to describe a free self avoiding walk chain. To study the properties of a microgel crosslinks have to be introduced. The most obvious statistical representation of a microgel is a self crosslinked single chain. Such a situation has been studied many years ago by the Manchester group in three papers [14]. The static properties have been computed by variational techniques. In this paper we choose a different route. Let us therefore consider a microgel as a self crosslinked polymer of roughly spherical structure, which can be visualized as given in Fig.(11).

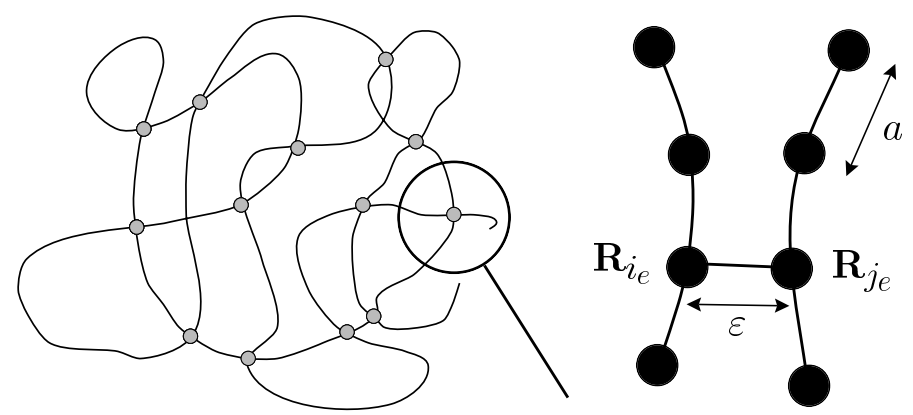

FIG. 1. A self crosslinked chain forming a microgel. The crosslinks are represented by distance constraints. Two segments are held a distance $\epsilon$ apart. The hard microgel is given by $\epsilon \rightarrow 0$.

In this paper we want to describe the crosslinks such that a continuous transition between the free chain and the fully crosslinked state can be represented in the same model. This has been motivated by our earlier work, where soft crosslinks have been introduced. The method of using soft crosslinks is able to interpolate from the free chain to the hard microgel. The state in between is a new kind of branched chain, whose scaling properties have been already described 15 18]. To be more precise let us introduce $M$ permanently crosslinked monomers, where each of them is characterized by pair of randomly chosen monomer coordinates $i_{e}, j_{e}$ that form a permanent crosslink. In fact, the whole set of crosslinks $\mathrm{C}=\left\{i_{e}, j_{e}\right\}_{e=1}^{M}$ determine the random connectivity of the micro network. These definitions and proposals allow us to formulate the partition function of the microgel, i.e.,

$$
Z(\mathrm{C})=\int_{V} \prod_{i=0}^{N} d \mathbf{R}_{i} e^{-\left(H_{W}+H_{I}\right)} \prod_{e=1}^{M} \delta\left(\mathbf{R}_{i_{e}}-\mathbf{R}_{j_{e}}\right)
$$

The partition function describes the Gaussian network with self avoiding interactions, and takes into account the total connectivity of the certain crosslink configuration C. If the delta constraint for the permanent crosslinks is represented by a soft Gaussian function, i.e., $\delta(x)=\lim _{\varepsilon \rightarrow 0}($ const $/ \epsilon) \exp \left(-\left(3 / \epsilon^{2}\right) x^{2}\right)$, the problem can be solved exactly. Thus 
we model the $\delta$-function by Gaussian distribution with width $\epsilon$ in limit $\epsilon \rightarrow 0$ and formulate the Hamiltonian of the crosslinked chain by

$$
\begin{aligned}
H_{W}= & \frac{3}{a^{2}} \sum_{i=1}^{N}\left(\mathbf{R}_{i}-\mathbf{R}_{i-1}\right)^{2}+\frac{3}{\epsilon^{2}} \sum_{e=1}^{N}\left(\mathbf{R}_{i_{e}}-\mathbf{R}_{j_{e}}\right)^{2} \\
& +v \sum_{0 \leq i \leq j}^{M} \delta\left(\mathbf{R}_{i}-\mathbf{R}_{j}\right)
\end{aligned}
$$

\section{MICROGELS WITHOUT INTERACTIONS}

Although we have shown in previous publications that the partition function can be solved for any value of $\epsilon$ exactly it is useful to rederive the results previously by the use of scaling arguments. These consideration will reproduce the exact results apart from prefactors. To do so it is useful to recall that the classical random walk contains two elastic contributions, one for stretching and one for compression. The addition of both yield a free energy $F \sim \frac{R^{2}}{a^{2} N}+\frac{a^{2} N}{R^{2}}$ and by minimization the size of Gaussian chain $R \simeq a N^{1 / 2}$ is recovered. Let us shortly repeat the results for later use. For the case of soft crosslinks, i.e., whenever $\epsilon$ is within the range $a^{2}<<\epsilon^{2}<<a^{2} N M$ we have for the relevant part of Flory free energy

$$
F \simeq \frac{N a^{2}}{R^{2}}+\frac{1}{\epsilon^{2}} M R^{2}
$$

and minimization yields the branched polymer regime

$$
R \simeq a\left(\frac{\epsilon}{a}\right)^{1 / 2}\left(\frac{N}{M}\right)^{1 / 4}
$$

The appearance of the typical branched polymer exponent $\nu=1 / 4$ is not surprising in the ideal case, since the constraint can be visualized as springs. Then the connectivity is changed. The branched polymer regime in the range of $a<<\epsilon<<a \sqrt{N M}$ was confirmed also by the exact solution of the crosslinked chain problem, see [16]. Moreover it agrees also with the corresponding branched polymer 18]

In the case of hard crosslinks, whenever $\epsilon \simeq 0$ or $a$ the crosslink term must be differently estimated. To do so the crosslink term can be estimated by the size of the random walk through crosslinks. Then the relevant part of Flory free energy is then given by

$$
F \simeq \frac{a^{2} N}{R^{2}}+M \frac{R^{2}}{a^{2}\left(\frac{N}{M}\right)}
$$

Minimization of the free energy provides the size of the microgel by

$$
R \simeq a\left(\frac{N}{M}\right)^{1 / 2}
$$

as also given by the exact results 16. Formally the latter result can be found from a special choice for $\epsilon$ from the corresponding result for soft gels (6), but note that the way of estimating the free energy contribution of the crosslinks is estimated very differently. Thus there can be a different prefactor, which is not accessible by scaling. The exact values for the radius of gyration of the non-interacting but crosslinked chains have been computed exactly in [16], where the numerical prefactors can be found.

\section{MICROGELS WITH EXCLUDED VOLUME}

Although we have been able to compute the size of the microgel exactly whenever the interactions are not present the self avoiding case appears very difficult. The only possibility for the problem at this stage is to use Flory estimates for size $R$. Let us first consider the case of soft crosslinks. The distance constraint that forces two arbitrarily chosen 
polymer segments together shrinks the chain. The shrinkage costs entropy penalty which balances with the distance constraint. The use of the pseudo potentials allows us, however, to cast this in a simple Flory free energy to

$$
F \simeq M \frac{R^{2}}{\epsilon^{2}}+a^{3} \frac{N^{2}}{R^{3}}
$$

and minimization yields the size of the swollen soft microgel

$$
R \simeq a\left(\frac{\epsilon}{a}\right)^{2 / 5}\left(\frac{N^{2}}{M}\right)^{1 / 5}
$$

The result is very intriguing. Although the Gaussian chain size scales the same way as the branched polymer, the swelling behavior produces another excluded volume exponent, resulting in a different swelling behavior as branched chains. In the latter case the swollen branched chain is characterized by $R \propto N^{1 / 2}$. On the other hand it can also be seen that the pure scaling in terms of the variable $N / M$ always present in the Gaussian case is destroyed. This becomes clear, since the excluded volume introduces interactions.

A similar estimate of the size can be carried out in the case of hard crosslinks. Here the elastic term stemming from the pseudo crosslink potential can be estimated the same way as in the case of non interacting networks, which was given by a random walk through the crosslinks. The total excluded volume energy remain the same, because it depends only on the total amount of monomers and not on the special connectivity. Then minimization of the free energy

$$
F \simeq M \frac{R^{2}}{a^{2}\left(\frac{N}{M}\right)}+a^{3} \frac{N^{2}}{R^{3}}
$$

yields swollen $c^{*}$-micro gels of size

$$
R \simeq a M^{1 / 5}\left(\frac{N}{M}\right)^{3 / 5}
$$

The size might appear small compared to what is expected intuitively. This comes from the fact that the crosslinks has been chosen totally randomly. In many theories of macroscopic networks the choice of the crosslink pairs is guided by the conformation of the excluded - or random walk chain, i.e., the often terminated "zeroth replica" [20]. For completeness we mention that the number of crosslinks in excluded volume gels cannot be arbitrarily large. A natural limit of the crosslink number is given by the condition that the size must be larger than a fully collapsed ball of space filling density, i.e. $R \geq a N^{1 / 3}$. The latter condition yields the upper limit for the number of crosslinks to be $M \leq N^{2 / 3}$.

\section{ADSORPTION BEHAVIOR OF SELF SIMILAR MICROGELS, GENERAL REMARKS}

Let us first study the adsorption of ideal microgels near a flat surface by naive scaling arguments. To do so, we repeat the scaling idea of de Gennes for arbitrarily flexible objects of arbitrary connectivity, but selfsimilarly linked. The connectivity of of such Gaussian fractal networks can be described by the spectral dimension $D$. The total number of monomers is therefore $N=m^{D}$, and their ideal size is given by $R_{0}=a m^{(2-D) / 2 D}=a N^{1 / d_{\mathrm{f}}}$, yielding a fractal dimension of $d_{\mathrm{f}}=2 D /(2-D)$. Thus $m$ counts the number of monomers through a linear dimension through the fractal object. This way of description includes the wellknown cases of linear chains for $D=1$ and for randomly branched polymers, i.e., $D=4 / 3$.

A simple way of looking at the adsorption conditions is to compare the free energy penalty of confinement of the Gaussian structure near the wall with the gain of energy by adsorbing a certain number of monomers.

$$
F \simeq F_{\text {conf }}-n_{a} w
$$

For Gaussian chains the confinement is simply given by by $F_{\text {conf }} \sim\left(R_{0} / H\right)^{2}$. Here we have used the symbol $H$ for the height of adsorbed layer, $n_{\mathrm{a}}$ is the number of adsorbed monomers, and $w$ is the gain of energy per $k_{B} T$. Following de Gennes book [19] we can reproduce the result given there for the linear ideal chain.

To do so we must first estimate the number of adsorbed monomers at the surface, we assume that the surface is penetrable for a moment. This yields immediately 


$$
n_{\mathrm{a}} \simeq a R^{2} \cdot \frac{m^{D}}{R^{2} H} \sim N\left(\frac{a}{H}\right)
$$

The main problem is to estimate the confinement free energy for Gaussian fractals with a larger connectivity compared to the linear chain. This is definitely not just the inverse of the Gaussian free energy of stretching, i.e., $F_{\text {stretch }}=$ $R^{2} / a m^{(2-D)}$, because upon stretching only the monomers in the shortest path are taking part on the deformation, whereas upon confinement the total number of monomers are concerned. The corresponding confinement free energy must then be of the form

$$
F_{\text {conf }} \cong \frac{N}{H^{d_{\mathrm{f}}}}
$$

This result contains the special case of linear chains, $D=1, d_{\mathrm{f}}=2$, and the latter agrees with the classical confinement free energy.

For the ideal Gaussian structures the height of the adsorbed layer scales as

$$
H \simeq a\left(\frac{1}{w}\right)^{1 /\left(d_{\mathrm{f}}-1\right)}
$$

The same result can be found by a blob argument. For selfsimilarly branched polymers a blob model can be used [5]. The manifold is confined to a height $H$. Inside blobs of diameter $H$ the branched chain is Gaussian, i.e., $H \simeq g^{(2-D) / 2 D} a$, where $g$ is the number of monomers inside the blob. Thus the number of blobs is given by $n_{\mathrm{b}} \simeq m^{D} / g=N / H^{2 D /(2-D)}$. The confinement free energy is proportional to the number of blobs, i.e., $F_{\text {conf }} \sim n_{\mathrm{b}}$. Employing the same scaling argument as above yields immediately

$$
H \propto a(1 / w)^{(2-D) /(3 D-2)}
$$

which agrees with the above result, eq.(16).

In any case, the above arguments only yield the behavior of ideal chains near the surface. For excluded volume chains and excluded volume manifolds the Gaussian elastic entropy penalty must be replaced by the confinement energy. To do so, the manifold can be put between two plates of distance $H$. This procedure yields similar results along to those derived in de Gennes book [19].

To do so, the extension of the manifold between two parallel plates must be computed. It is given by $R_{\|} \simeq$ $H(a / H)^{5 / 4} N^{(2+D) / 4 D}[5]$. This result is consistent with the linear SAW chain between two plates. For $D=1$ the two dimensional chain of blobs is recovered. The confinement free energy can be only a function of the ration of the size of the manifold and the distance between the plates, i.e., $(R / H)$, where $R$ is the size of the self avoiding manifold $R \simeq a N^{(2+D) / 5 D}$. The confinement free energy is then easily found from the condition that the free energy must be an extensive quantity. Thus it must scale as $F_{\text {conf }} \propto N$, which yields

$$
F_{\text {conf }} \simeq k T\left(\frac{a}{H}\right)^{5 D /(2+D)} N
$$

Replacing the elastic free energy in the scaling argument above by the confinement energy, yields the physically sensible result

$$
H \propto a w^{-\frac{2+D}{4 D-2}}
$$

which is now independent of the molecular weight for any manifold. Moreover for $D=1$ the linear chain result is recovered. Moreover the result bears an interesting point in it: The parameter $w$ is the gain of energy at adsorption of one segment per thermal energy $k T$. Thus it is physically reasonable that this parameter is sufficiently small, i.e., $w<1$. Thus a significant change of the behavior can be expected if the exponent in $H$ is larger and smaller than one. It is interesting to note that this happens at $D=4 / 3$ which is close to the spectral dimension of randomly branched chains or accidentally for percolation clusters. Thus randomly connected manifolds of large connectivity adsorb weaker than objects of low connectivity, as linear chains. This is physically intuitively clear since the number of accessible sites become smaller for increasing connectivity. In the following section we will use the same strategy to discuss the adsorption behavior of microgels.

\section{CONFINEMENT ENERGY FOR MICROGELS WITH EXCLUDED VOLUME}

As we have seen in the last paragraph on the previous section we have to construct the free energy of confinement. We use the same concept of gels between two plates for energy cost of squeezing. We have seen in the first two sections 
that we can distinguish between soft and hard microgels by the value of the parameter $\epsilon$. For both cases we expect physically different behavior.

Let us first consider soft gels between plates. To do so we have to determine the size of the gel parallel to the plates. The relevant parts of the free energy is given by

$$
F \simeq M \frac{R_{\|}^{2}}{\epsilon^{2}}+a^{3} \frac{N^{2}}{H R_{\|}^{2}}
$$

where we have argued that the elastic confinement and the "anisotropic" excluded volume term balance each other. This yields immediately

$$
R_{\|} \simeq H\left(\frac{\epsilon^{2} a^{3}}{H^{5}}\right)^{1 / 4}\left(\frac{N^{2}}{M}\right)^{1 / 4}
$$

and the corresponding confinement energy to

$$
F_{\mathrm{conf}} \simeq \frac{N}{\sqrt{M}} \frac{\epsilon}{a}\left(\frac{a}{H}\right)^{5 / 2}
$$

Note that the confinement free energy is determined by the fact that it must be proportional to the total number of monomers, since the free energy is extensive.

The same procedure can be employed for hard gels. The relevant free energy takes a similar form as before, apart from the elastic part of the energy.

$$
F \simeq \frac{M^{2} R_{\|}^{2}}{a^{2} N}+a^{3} \frac{N^{2}}{H R_{\|}^{2}}
$$

The size of the gel parallel to the plates is given for completeness. It scales as $R_{\|} \simeq H(a / H)^{3 / 4}\left(N^{3} / M^{2}\right)^{1 / 4}$. To find the confinement free energy the same argument yields

$$
F_{\mathrm{conf}} \simeq\left(\frac{a}{H}\right)^{5 / 3} \frac{N}{M^{2 / 3}}
$$

An important observation is that for $M=1$ (no crosslink) linear chain is recovered.

\section{ADSORPTION BEHAVIOR}

Finally we are in the position to discuss the adsorption behavior of the gels. To begin with, we employ the same scaling arguments as given in the case of self-similar polymeric fractal. Thus we have to consider the competition between confinement, or the entropy penalty of confinement and the energy gain by adsorption. This results in a total free energy of the general form

$$
F \simeq F_{\mathrm{conf}}-n_{a} w
$$

We just summarize the results to be brief. First for soft gels we find

$$
H \simeq a w^{-2 / 3}\left(\frac{\epsilon}{a}\right)^{2 / 3} M^{-1 / 3}
$$

Similarly for hard gels

$$
H \simeq a w^{-3 / 2} M^{-1}
$$

Note that the latter equation contains the free chain result for $M=1$, i.e., if no crosslinks are present. We see that in both cases the height of the adsorbed layer depends on the number of crosslinks in a significant and characteristic way. The results are in accordance with the physical intuition. The soft microgels adsorb more easily, because these objects are more flexible. This is also shown by the different exponents of the interaction energy $w$. 


\section{SUMMARY AND CONCLUSION}

With use of Flory-Approximation we had investigated behavior of microgels. The complexity of the distribution function of the non interacting network prevented us to use more refined methods, as they are well known in the case of linear polymer chains. Nevertheless we got results which are reasonable and could be checked by experimental methods, at least in their tendency. The cases worked out here have been relevant for penetrable surfaces, i.e., interfaces. A direct comparison to hard surfaces is not possible, since the number of monomers close to the surface cannot be determined by eq. (14). The case of linear chains in half space has been studied in detail, see e.g. [1] for a general reference. Crossover exponents and new critical points determine the physics. In the present case of microgels and polymeric manifolds a similar treatment appears very difficult, since the "bare propagator" has a very complicated structure, although it is exactly known [15,16]. Nevertheless we expect that the principal statements can be compared at least qualitatively with experiments.

To study the adsorption behavior we first had to calculate the size of the microgel in solution. This has been carried out by employing the Flory arguments. The basis for the reliability of the result has been their agreement withe comparison of exact calculations without excluded volume in bulk. Then generalization opened the determination of sizes of microgels with excluded volume interaction in bulk systems and near adsorbing flat surfaces. Moreover we made straightforward generalizations to fractal type microgels, which could be described by the spectral dimension.

The results show a transition from polymeric type of adsorption behavior for soft microgels, i.e., with small number of crosslinks, or alternatively low spectral dimension, to colloidal adsorption, whenever the crosslink number is large and their coupling is strong.

We have seen that the results have been presented from considerations on single gels. This has also experimental interest on surface protection. A layer of adsorbed gels of height $H$ at a surface is a single gel problem. Unlike linear polymer chains the gels can no longer interpenetrate each other. Thus microgels and branched polymers appear more effective in surface protection.

Another interesting question is also the interplay between vulcanization and adsorption. In the case we studied so far, we had assumed preformed gels and fractals which had then brought to the interacting surfaces. The other case, i.e., the vulcanization in presence of interacting walls would lead to new types of gels with new structures which depend on the strength of the surface - monomer interaction [21,22].

\section{ACKNOWLEDGMENT}

L'un des auteurs (TAV) remercie Pierre - Gilles de Gennes et Elie Raphaël pour leurs remarques interessantes sur ce travail. Nous remercions aussi l'un des critiques pour avoir attiré notre attention sur une methode élégante d'obtantion du "blob", qui a permis d'ameliorer la qualité de ce papier.

[1] E. Eisenriegler, Polymers at surfaces, World Scientific, Singapore, 1994

[2] P.G. de Gennes, Simple views on condensed matter, World Scientific, Singapore, 1992

[3] J. Rühe, preprint, 1997

[4] T.A. Vilgis, P. Haronska, Macromol. Theory Simul., 4, 111, (1995)

[5] T.A. Vilgis, P. Haronska, M. Benhamou, J. Phys.II, 4, 2139, 1994

[6] E. Zhulina, T.A. Vilgis, Macromolecules, 28, 1008, (1995)

[7] M. Antonietti, D. Ehrlich, K.J. Fölsch, H. Sillescu, M. Schmidt, P. Lindner, Macromolecules, 22 (1989) 2802

[8] M. Antonietti, K.J. Fölsch, H. Sillescu, T. Pakula, Macromolecules, 22 (1989) 2812

[9] M. Antonietti, W. Bremser, K.J. Fölsch, H. Sillescu, Makromol. Chem., Makromol. Symp., 30 (1989) 81

[10] M. Antonietti,Ch. Rosenauer, Macromolecules, 24 (1991) 3434

[11] T.A. Vilgis, Physica A 153, 341, (1988)

[12] P. Haronska, T.A. Vilgis, J. Chem. Phys., 102, 6586, (1995)

[13] I. Carmesin, K. Kremer, J. Phys. (France), 51, 915, (1990)

[14] G.Allen, J. Burgess, S.F. Edwards, D.J. Walsh, Proc. R. Soc. Lond. A, 334, 453, 465, 477, (1973)

[15] M.P. Solf, T.A. Vilgis, J. Phys. A, 28, 6655, (1995)

[16] M.P. Solf, T.A. Vilgis, J. Phys. I (France), 6, 1451, (1996)

[17] M.P. Solf, T.A. Vilgis, Phys. Rev. E, 55, 3037, (1997) 
[18] T.A. Vilgis, Macromol. Theory Simul., in press, (1997)

[19] P.G. de Gennes, Scaling concepts in polymer physics, Cornell University Press, Ithaca, 1979

[20] R. T. Deam, S.F. Edwards, Proc. Trans. R. Soc. London. A 260, 31, (1976)

[21] M.A. Cohen Stuart, J.T.F. Keurentjes, B.C. Bonekamp and J.G.E.M. Fraaye, Colloids Surfaces 17, 91, (1986)

[22] M. Abel, R. Lipowsky, unpublished 